\title{
A Reconfigurable OADM Architecture for High-Order Regular- and Offset- QAM based OFDM Super-Channels
}

\author{
Kyriakos Vlachos, Filipe Ferreira and Stylianos Sygletos
}

\begin{abstract}
We investigate the performance of a Reconfigurable All-Optical Add Drop Multiplexer (ROADM) architecture, that is suitable of supporting high-order regular and offset based QAM signals. The design is based on a highly modular interferometric, structure, in which OFDMA sub-channels remain in the optical domain. Sub-channel extraction is achieved via waveform splitting and coherent subtraction. Numerical studies have been carried out to validate performance and optimize critical parameters of the add/drop process. Particularly, input parameters like the signal extinction ratio, pulse rise time, switching window and input power levels have been optimized for single sub-carrier add/drop operation, from an OFDM super-channel comprising seven optical sub-carriers with $10 \mathrm{GHz}$ spacing. Results have shown that the proposed ROADM architecture can accommodate and process both regular and offset based high-order QAM signals.
\end{abstract}

Index Terms-Add-Drop multiplexing, QAM signals, optical superchannel, OFDMA, coherent detection.

\section{INTRODUCTION}

$\mathrm{R}$ econfigurable add-drop multiplexers (ROADMs) play a crucial role in optical networking, as they enable transparent demultiplexing, routing, and generally processing (i.e. adding/dropping) of the wavelength channels in the optical domain [1]. Since their early development, ROADMs have been evolved from plain wavelength blockers to multi-degree wavelength selective switches and are widely deployed to confer better bandwidth utilization and wavelength management to network administrators, as well as easy monitoring and planning functionalities. Classic ROADMs, particularly those that are using directional Wavelength Selective Switches (WSS), target dense wavelength division multiplexing (DWDM) transmission systems for typical

Manuscript received day month year, revised day month year, accepted day month year. Date of publication day month year, date of current version day month year. This work has been partially supported by the EU-ICT project FOX-C (grant number 318415) and by the Engineering and Physical Sciences Research Council (EPSRC) through PEACE (EP/L000091/1) and TRANSNET (EP/R035342/1) projects.

Kyriakos Vlachos is with the Computer Engineering and Informatics Department, University of Patras, GR26500, Rio, Greece (email: kvlachos@ceid.upatras.gr).

Stylianos Sygletos and Filipe Ferreira are with the Aston Institute of Photonic Technologies, School of Engineering and Applied Science, B4 7ET, Birmingham, UK (email: s.sygletos@aston.ac.uk,f.ferreira@aston.ac.uk) add/drop operations of the employed wavelength channels. Typically, there are fixed wavelength assignments to specific ports and fixed direction assignments for (de)multiplexers. Dropped channels create empty spectral slots for new (same wavelength) DWDM channels to be added. In this way, transparent links are dynamically created, avoiding any electronics processing between the link edges, thus enabling bandwidth tuning, by adding or dropping extra wavelengths.

With the recent introduction of highly spectral efficient multiplexing technologies in optical transmission, such as, orthogonal frequency division multiplexing (OFDM) [2],[3] and Nyquist-WDM [4],[5] the traditional ROADM solutions became insufficient. For the case of Nyquist-WDM signals, extremely sharp optical pass-band filters will be required to perform add-drop multiplexing at sub-channel level with limited guard band interval [6],[7]. For OFDM signals, such pass-band filtering is inappropriate due to the spectral overlapping of the neighboring sub-channels, requiring instead the use of advanced interferometric schemes [8],[14]. This is because, orthogonality between OFDM channels must be pertained for proper demultiplexing, without inter-channel crosstalk. This poses stringent requirements in the generation of the actual optical sub-channels, especially at high baud-rates, as it mandates the use of orthogonal pulses. This however is not impossible due to the limitations of today's electrical equipment $(50 \mathrm{GHz})$. Toward this end, it has been shown, through numerical simulations in [8], that with the use of an optoelectronic interferometric structure, employing digital coherent detection, (Nyquist or OFDM) sub-channel add/drop operation can be achieved. Combining an opto-electronic interferometer, supported by off-line digital signal processing (DSP), and a highly nonlinear fiber to enhance the optical field superposition, single-carrier add/drop multiplexing has been also experimentally demonstrated from a 3-carrier OFDM super-channel, [9], with up to 32-QAM modulated signals, [10], and with PDM tributaries, [11]. Despite this first, non-real time demonstration, it is clear that the use of digital coherent detection in one of the interferometer branches is prohibitive for high-speed and high-scalable systems, making all-optical solutions a much preferable option.

Whilst some progress has been made towards this goal by allowing spectral guard bands between multi-banded OFDM signals [12], [13], with sub-channel spectral overlapping, however conventional ROADMs technologies are unable to 

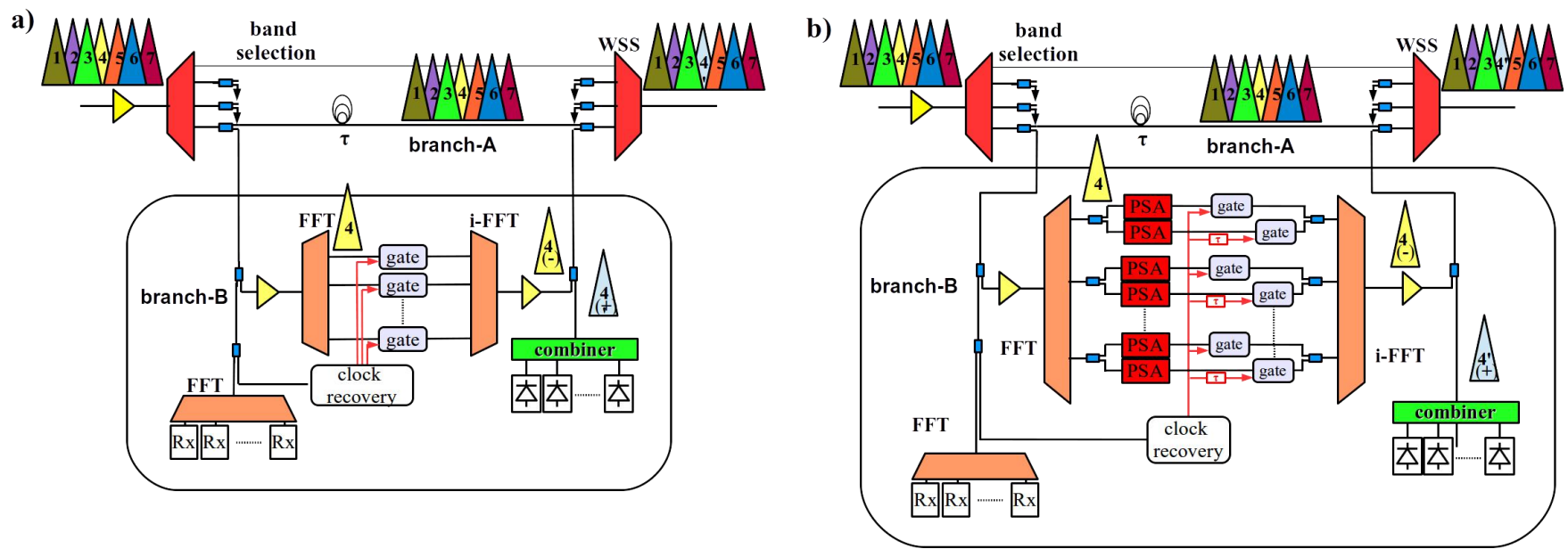

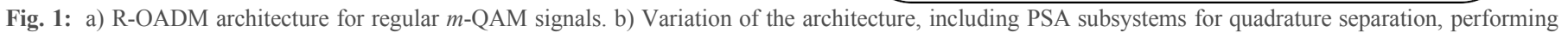
asynchronous sampling on the two quadrature components.

perform add/drop wavelength processing without violating the signal orthogonality condition that ensures crosstalk free performance. Thus, unless there will be a sacrifice in spectral efficiency, through the use of guard-band intervals, the inter-symbol interference (ISI) induced crosstalk from the sub-channel multiplexing process will limit the scalability of the system to low constellation orders [14]. In [16], it has been shown that by offsetting the in-phase and quadrature tributaries by half the symbol period in time, the ISI crosstalk can be eliminated even using practical signal spectral profiles or pulse shapes. This may enhance spectral efficiency, employing high-order offset-QAM, with performance approaching the theoretical limits.

In this paper, we investigate the performance and extend the operation of a recently proposed ROADM architecture,[14],[15] that is capable of supporting high-order regular- as well as offset- QAM signals. This is accomplished by employing a nonlinear phase sensitive operation process, which allows separating the in-phase from the quadrature component of the replicated channel. The architecture is based on linear all-optical methods, such as FFT/i-FFT filtering and time domain sampling, for creating a replica of the sub-channel signal, a copy of which is detected locally for the dropping process. The node is completed using a simple coherent insertion process, of the new channels. Through an in-depth numerical analysis, the main degradation causes have been identified, while critical parameters for channel insertion/extraction have been optimized.

The rest of the paper is organized as follows. Section II presents the ROADM architecture, while Section III presents numerical results organized in three sub-sections, with respect to sub-channel specifications (guard-bands, pulse rise times etc), sub-channel add/drop multiplexing performance and the effect of the FFT/i-FFT resolution. Section IV summarize findings and draws conclusions on the ROADM design and its performance.

\section{ROADM ARCHITECTURAL DESIGN}

The ROADM architecture for regular QAM signals, initially proposed in [14] and implemented in [17] is depicted in Figure 1a. The modified architecture for the processing of offset-QAM signals, is achieved by introducing a band of Phase Sensitive Amplifiers (PSAs), and is shown for the first time in Figure 1b. The PSA sub-system is commissioned to amplify the in-phase and suppress the quadrature components of the input signals. In both schemes, at the node input, the selected super-channel is split into three copies. One copy is dropped to the local receivers and the two remaining copies feed the interferometer branches-A and -B. It should be noted that in a complete network scenario, (i.e. ROADMs' cascaded performance in a metropolitan network), any residual dispersion should be compensated, before OFDM signals enter the node.

Two conventional wavelength selective switch (WSS) units, one at the input and one at the output of the node, perform the selection of the super-channel and its re-insertion to the transmission link after the sub-channel add/drop multiplexing. Both WSSs should have a capability for grid-less operation. Within the super-channel to be processed, sub-channel switching is achieved by means of a two-branch modular interferometer structure, denoted as Branch-A and Branch-B (see Figure 1a and Figure 1b). The architecture is modular in the sense that additional interferometer sub-paths can be connected in parallel using the available WSS ports to increase the number of super-channels that can be simultaneously processed by the node.

Sub-channel blocking is facilitated by replicating the corresponding signal waveform in branch-B and interfering it destructively with the super-channel that propagates through branch-A. During the channel replication process, the channels remain in the optical domain, while they are processed in the following three stages. The first stage involves de-multiplexing of the OFDM signal by an optical Fast Fourier Transform (FFT) processor [18],[19]. For the architecture addressing regular m-QAM signals (see Figure 1a), a subsequent bank of optical gates [20],[21], one for each sub-channel, performs time sampling of the sub-channels that need to be blocked from the path. The gates are synchronized to a common clock signal that is extracted from the super-channel receivers. Each one of them 

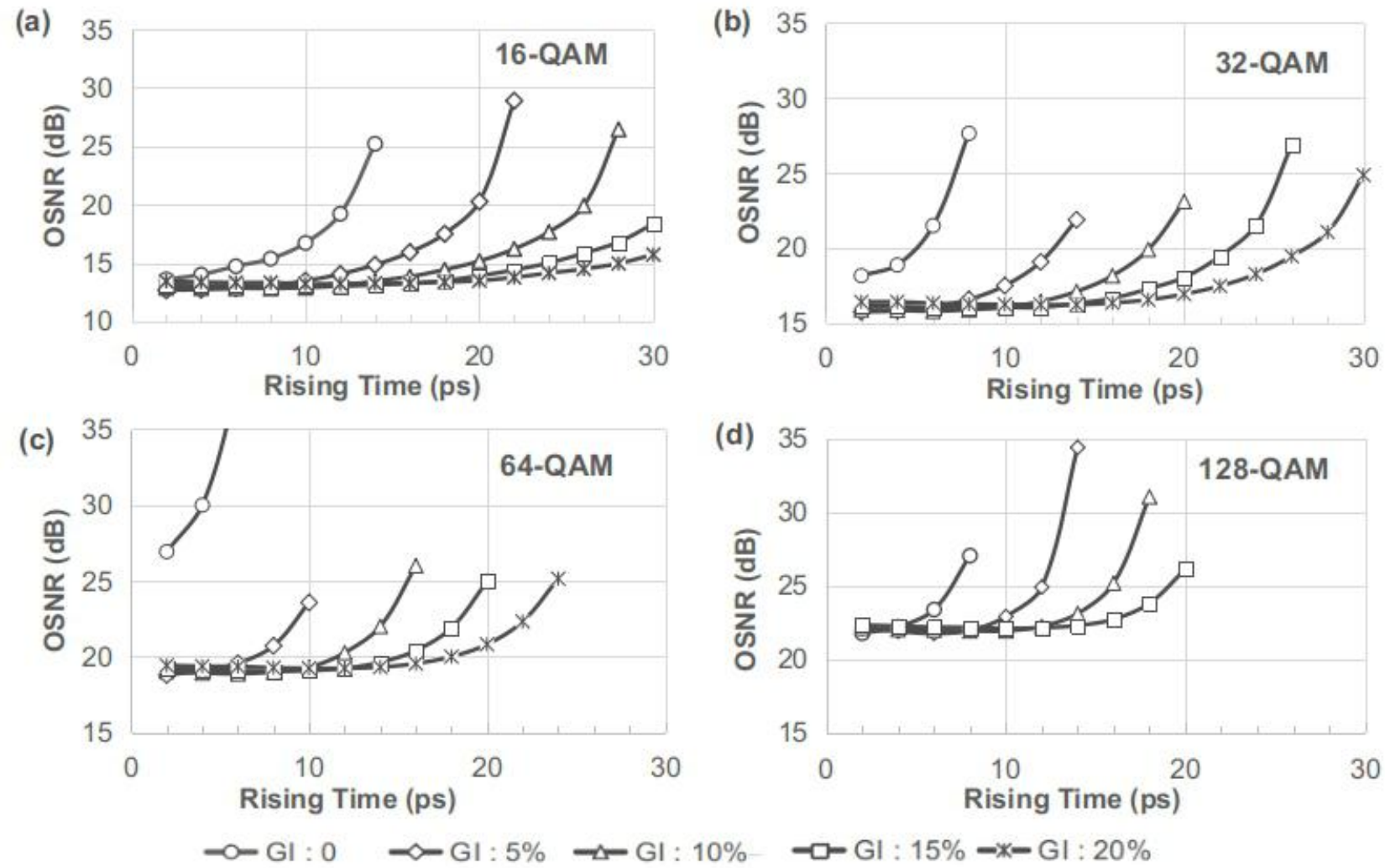

Fig. 2: Back-to-back performance of an optical OFDM super channel that consists of seven sub-channels, each carrying regular m-QAM modulation at 10 GBaud. The results depict OSNR levels at the receiver that correspond to a BER of $10^{-3}$, as a function of the pulse rise-time and guard-band intervals (GI), for the constellation orders of a) $m=16$, b) $m=32$, c) $m=64$ and d) $m=128$.

selects a sampling window of minimum crosstalk and feeds the waveform to an optical i-FFT processor, with transfer function $\mathrm{H}(\mathrm{f})=\operatorname{sinc}(\mathrm{fT})$, which reshapes the pulses back to their initial symbol duration T. Although the use of ideal rectangular pulses is not experimentally efficient, however, the important parameter here, is to employ an adequate narrow pulse to achieve good reconstruction rather than more feasible pulse shapes.

For the case of offset-QAM signals (see Figure 1b), the architecture is modified by including a set of phase sensitive amplifiers, i.e. one PSA for each demultiplexed sub-channel. These are commissioned to separate signal's quadrature components. Each PSA has also the capability of suppressing phase distortions that originate from the in-line amplifiers, as well as, Kerr-based nonlinear effects in the transmission link. The necessary bank of optical gates, is located at the output of each PSA. In the case of offset QAM signals, the associated switching windows are characterized by a fixed delay difference of $T / 2$. The in-phase and quadrature components of the signal are subsequently recombined and directed to the i-FFT processor, where pulse reconstruction takes place.

Subsequently, for both schemes, the recovered waveform is amplified and interfered destructively with the OFDM signal coming from Branch-A. From this process, a spectral hole is created leaving an empty position for the new (sub-carrier) channel to be added. All losses, optical delays and relative optical pulses should be carefully adjusted to maximize the extinction of the channel extraction. The insertion of the new channels takes place separately, with a bank of laser transmitters aligned to the sub-channel frequencies of the OFDM super-channel.

\section{NUMERICAL ANALYSIS}

We have performed extensive numerical simulations to evaluate the performance of the node. For this, we have considered an OFDM super-channel comprising seven optical sub-carriers spaced at $10 \mathrm{GHz}$. The sub-carriers were modulated, according to the simulation scenario under study, either in regular or offset m-QAM format, from randomly generated binary sequences of rectangular pulses (1's and 0 's of equal probability). The electrical signals experienced a low-pass response from a $1^{\text {st }}$ - order Gaussian filter, giving them a rise time, which was considered one of the parameters under evaluation. For the case of regular m-QAM signals, a frequency guard interval (GI), expressed as a percentage of the sub-carrier spacing to the baud-rate, was also added between the OFDM sub-channels for increasing the signal robustness against any residual ISI induced crosstalk. For offset-QAM based OFDM signals the use of GI in not applicable [16]. The WSS units that performed the super-channel selection were simulated with a 3rd order Gaussian transfer function of $100 \mathrm{GHz}$ with $3 \mathrm{~dB}$ bandwidth. For the FFT/i-FFT filter we 

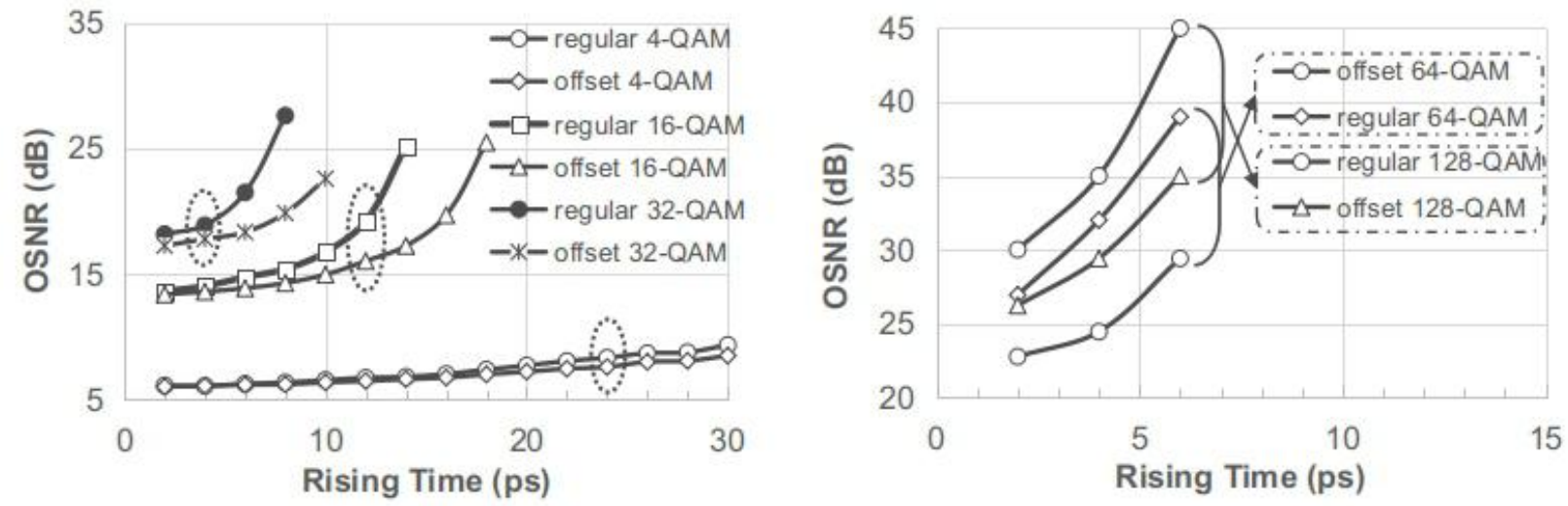

Fig. 3: Back-to-back performance of optical OFDM super channel with seven offset m-QAM modulated sub-channels at 10 GBaud. The results depict OSNR levels at the receiver that correspond to BER of $10^{-3}$, as a function of the pulse rise-time for different constellation order $m$ : (left) $m=\{4,16,32\}$ and (right) $m=\{64,128\}$.

considered the response of ideal devices, i.e. of $\sim \operatorname{sinc}(\mathrm{fT})$ shaped frequency transfer function, as well as, the response of more realistic LcoS based photonic spectral processors [22], [23]. Furthermore, in our study we have assumed that the optical gates create rectangular switching windows, synchronized to the demultiplexed pulse-streams, for which their time duration and contrast ratio could be independently varied. The optical amplifiers within the node had a noise figure equal to $4.5 \mathrm{~dB}$. Signals were detected using an optically pre-amplified coherent receiver with a zero-linewidth local oscillator (to allow us to focus on the impact of the node) and an FFT optical demultiplexing filter. Performance was quantified by an iterative Monte-Carlo method with direct error counting. Iterations were performed using each time different data patterns of $2^{15}-1$ bit length for the OFDM super-channels and they were terminated when the BER estimation was statistically expected to have less than $10 \%$ error [24].

\section{A. Transmitter performance for high order regular- and offset- QAM signals}

Figure 2 depicts the impact on the received OSNR level that corresponds to a BER of $10^{-3}$, as a function of the pulse risetime for different constellation orders $\mathrm{m}=\{16,32,64,128\}$ for regular m-QAM modulation of each sub-channel in the OFDM super-channel. As the rise time increases, significant ISI crosstalk is introduced that degrades the signal performance. High constellation order signals are more vulnerable to this type of degradation. The introduction of guard interval alleviates the impact of crosstalk and allows migration to high constellation orders using commercially available transmittes. For example, a typical transmitter that generates optical pulses of $\sim 15$ ps rise-time, may enable operation even for 128-QAM modulated sub-channels, when a $20 \%$ guard interval is used.

Next, we analyze performance of offset-QAM formats, for the same pulse rise-time values and perform a direct comparison with the regular QAM case. The impact on the received OSNR level for offset $\mathrm{m}$-QAM with $\mathrm{m}=\{4,16,32\}$ is depicted in Figure 3a, and for $\mathrm{m}=\{64,128\}$ in Figure 3b. As offset-QAM signals cannot operate with guard-bands in OFDM super-channels, direct performance comparison with regular
QAM signals is achieved for zero guard-band interval. The results show that using offset-QAMs gives superior performance, when scaling to large constellation orders. The net gain in OSNR span from $1 \mathrm{~dB}$ to $2 \mathrm{~dB}$ for a pulse rise time below $5 \mathrm{ps}$ and to more than $3 \mathrm{~dB}$ for higher rise times. The gain in performance depends on the modulation order, i.e. the higher order QAMs, the higher the OSNR gain becomes. In particular, for a pulse rise-time of $6 \mathrm{ps}$, the OSNR net gain using an offset rises from $0.1 \mathrm{~dB}$ to $0.9 \mathrm{db}$, to $3.1 \mathrm{~dB}, 9.6 \mathrm{~dB}$ and $10 \mathrm{~dB}$ respectively for $\mathrm{m}=\{4,16,32,64$ and 128$\}$ QAM. In what follows, 128-QAM format is omitted due to its relative poor performance.

\section{B. Sub-channel add/drop multiplexing performance}

The next step was to investigate the add/drop multiplexing performance of the node. For that, we considered drop, extract and insert operation of a single sub-channel (i.e. Channel 4) from the OFDM super-channel stream. Our goal was to identify the impact of the main physical limitations and to specify the optimum parameters of the associated devices and subsystems within the ROADM architecture. The first optimization concerned the duty cycle $\alpha$ of the gating window. The gating action combined with the i-FFT induced pulse shaping introduce additional losses in the parallel arm (i.e. Branch-B) for either of the two design (see Figure 1a and Figure 1b). A short switching window would allow a better reconstruction of the sub-channel pulses after the i-FFT filter, but at the expense of a significant OSNR degradation, since the losses of the sampling process would scale proportionally to $\alpha^{2}$. Thus, in our 10 Gbaud system, a $10 \%$ duty cycle corresponds to a switching window duration of $10 \mathrm{ps}$ and $20 \mathrm{~dB}$ effective losses.

The simulated pulses of each generated sub-channel had a rise-time of $2 \mathrm{ps}$, whereas the contrast ratio of the optical gates in the design was well above $30 \mathrm{~dB}$. In addition, ideal i-FFT/FFT filtering was considered. Following the optimization of [14], the optical couplers in the architecture were selected to have a loss of $3 \mathrm{~dB}$, whereas the loss of each WSS, optical gate and FFT/i-FFT filter $6 \mathrm{~dB}$. We have numerically investigated the trade-off in performance between the OSNR degradation that occurs in Branch-B and the 

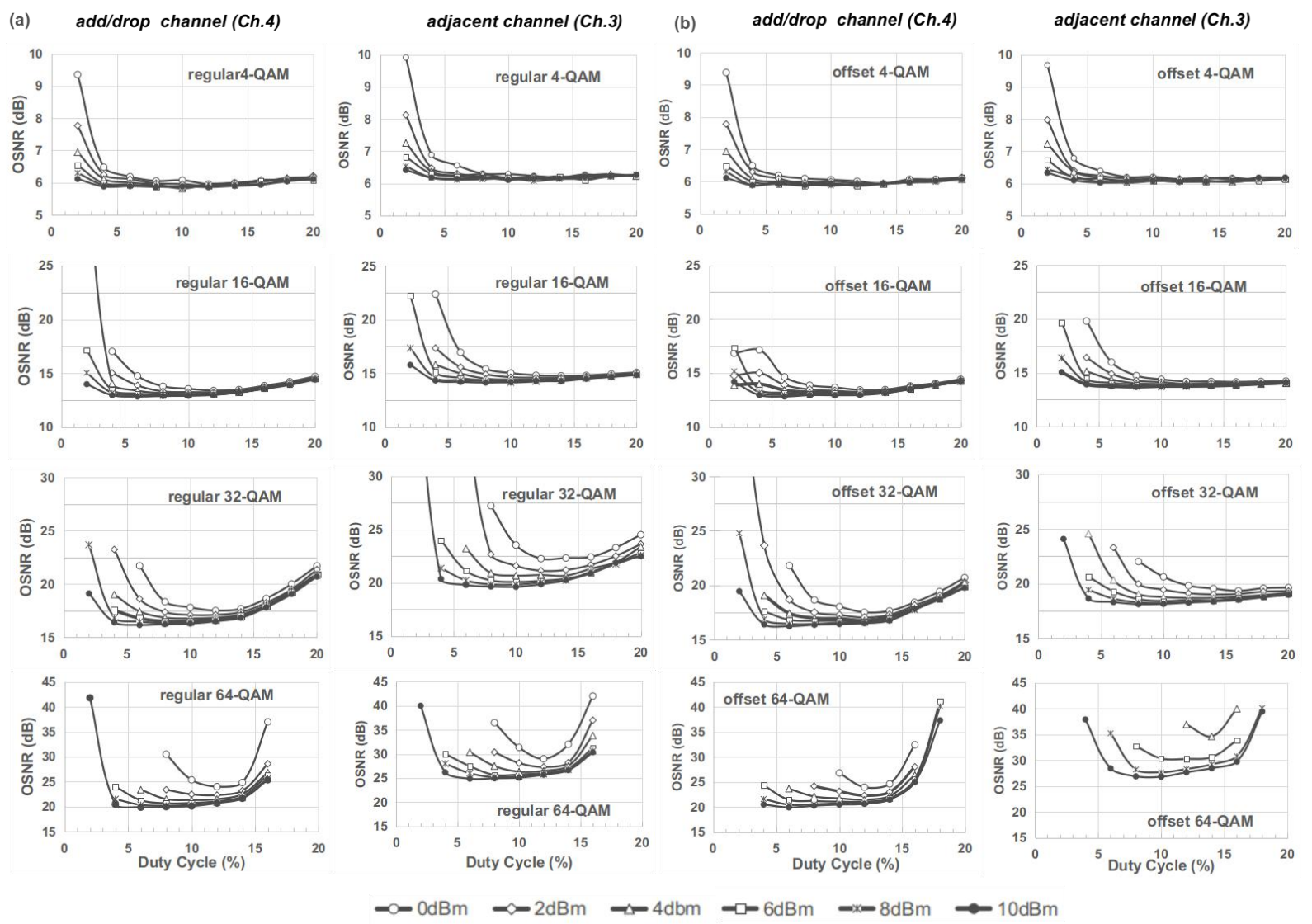

Fig. 4: (left) Regular and (right) offset high-order QAM signal, required OSNR loading at the receiver for achieving BER: $10^{-3}$ as a function of the switching window duty-cycle for different power levels at the input of the gate. The results have been taken for different constellation orders of the add-drop channel-4 and its closest neighboring channel 3 , which passes through the node.

inter-symbol interference introduced by the gating process. Figure 4 displays the relevant results, and in particular, the required OSNR loading at the receiver for achieving $\mathrm{BER}=10^{-3}$ as a function of the switching window duty-cycle for different power levels at the input of the gate. Numerical studies were carried out for both regular (see Figure4 left) and offset (Figure 4 right) QAM formats, and for different constellation orders $(\mathrm{m}=\{4,16,32,64\})$ of the add/drop channel (Ch. 4) and its closest neighbour (Ch.3), which passes through the node. From Figure 4, it is clear that low duty cycles result to an increase of OSNR degradation and in same cases in an extreme degradation.

For the case of regular 4-QAM, we notice that duty cycles above $5 \%$ had little impact on the add/drop performance. In contrast, for duty cycles less than $5 \%$, a rapid increase of the penalty can be seen due to the high OSNR degradation in the parallel path of the node. However, this was for low levels of the launched power. Increasing the power level, e.g. to $10 \mathrm{dBm}$, the effective noise figure of Branch-B improves and the corresponding OSNR degradation is significantly reduced. We also noticed that the performance of Ch. 4 was slightly better than that of Ch. 3 .

Going to the regular 16-QAM format, the operating margins were reduced. In this case, the operating switching window becomes significantly shorter with an optimum region identified around $8 \%$, i.e. 8 ps. At reduced duty-cycles (i.e. $<5 \%$ ), increasing the launched power to the gate had less impact on the performance improvement, whereas going to the side of high duty-cycles, we noticed strong influence of the residual ISI crosstalk passing through the incomplete sampling process. Moving to even higher constellation orders the main findings remain the same. However, we noticed a slight shift in the optimum switching window duration, towards larger values of duty-cycle i.e. towards $12 \%$. Another interesting point that becomes more apparent now is that the performance penalty was significantly higher for the neighboring Ch. 3 that passed through the node, than that of Ch.4, that experienced the add/drop operation. This is because, identical signal reconstruction in the parallel branch cannot be achieved, which would allow complete removal of the dropped signal. For 32-QAM signals, the OSNR penalty, between these two channels was approximately $4 \mathrm{~dB}$.

Results for the offset-QAM signals are shown in Figure 4-right, where again we have measured the required OSNR loading at the receiver for BER of $10^{-3}$. The main conclusions remain the same as with the case of regular QAM signals. At low duty-cycle values, a large penalty is noticed, that is due to the heavy OSNR degradation in the parallel branch of the 

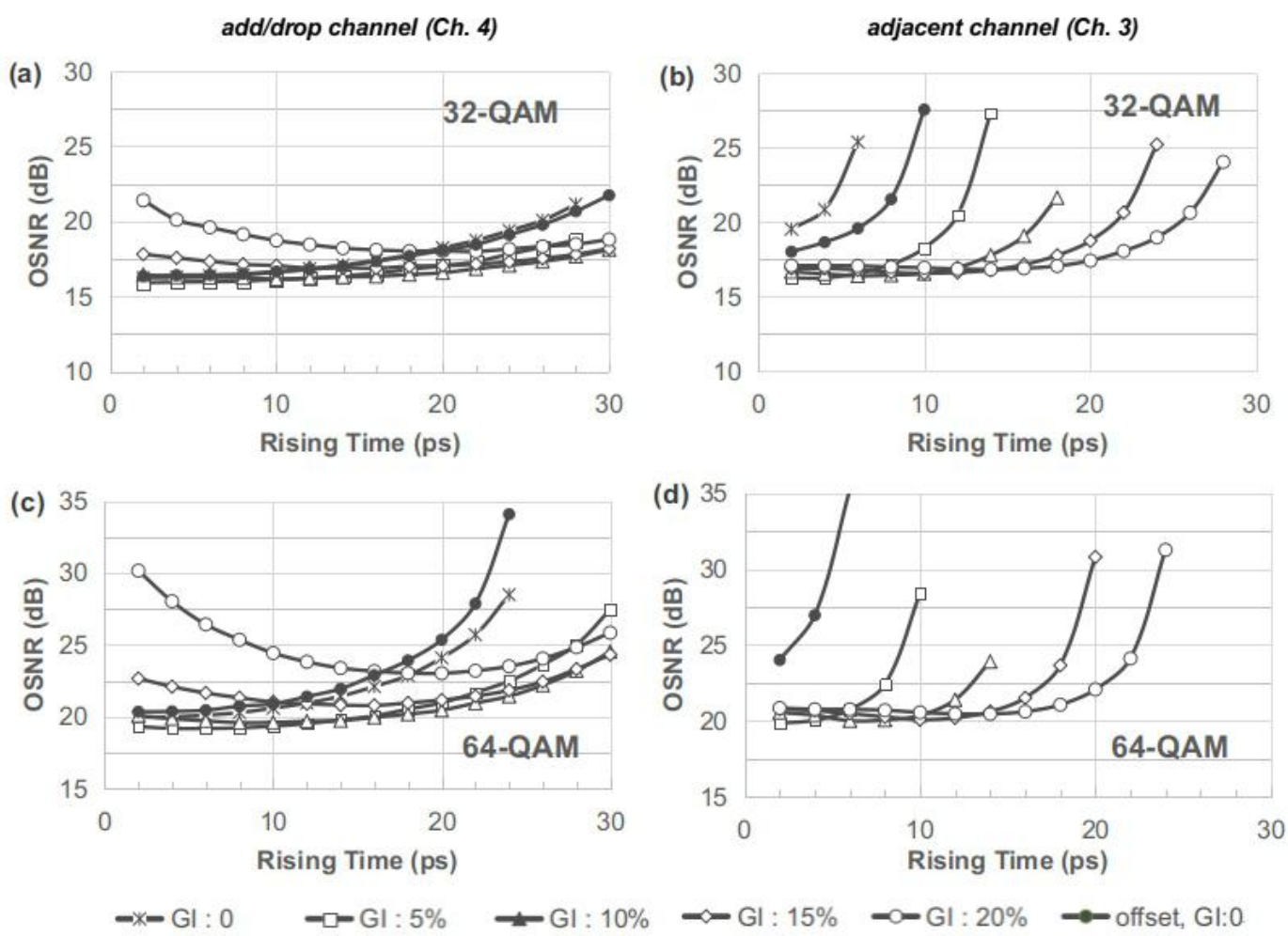

Fig. 5: Effect of rising time in required OSNR at the receiver for achieving BER: $10^{-3}$ for different constellation high-order (32 and 64-QAM) signal of the add/drop Channel 4 and its closest neighboring Channel 3. Results have been taken for different guard interval for both the regular as well as the offset QAM based modulation format.

architecture (i.e. branch-B). Again, this penalty can be mitigated for low constellation orders, increasing the launched power level at the optical gate input. Also, for constellations orders higher than 16-QAM, the incomplete sampling that occurred, when increasing the duty-cycle of the switching window, had a significant impact on the overall performance. The only difference that we noticed, when comparing the regular QAM case, is that with offset-QAM, there was a slightly lower induced degradation on the neighboring channel (Ch. 3).

Subsequently, we study the impact of pulse rise-time on the add/drop multiplexing performance of the node. For this analysis we considered many of the parameters of the node optimized, according to the previous results. Specifically, we considered a $10 \mathrm{dBm}$ power level at the input of the gate and a $10 \%$ duty cycle of the switching window. In Figure 5, we show the results of the required OSNR load at the receiving end (i.e. at the output of the node), which corresponded to $\mathrm{BER}=10^{-3}$, as a function of the rise-time of the pulses and the guard-band interval. The calculations have been carried out for both the add/drop channel (Ch. 4), as well as, for its closest neighbor (Ch. 3). Results only for the 32- and 64-QAM formats are shown here. For the cases of 4- and 16-QAM, the OSNR variation was negligible for rise times up to $25 \mathrm{psec}$. Particularly, 4-QAM signals required an OSNR level of $6 \mathrm{~dB}$, while 16-QAM signals required an OSNR level of $12 \mathrm{~dB}$, irrespectively of the pulse rise time. From Figure 5(a), we may see that increasing the pulse rise-time, a penalty is introduced due to the ISI crosstalk. It is surprising, however, that having a guard intervals (GI) the ISI effect is not alleviated. We also notice that for each constellation order, there is an optimum GI value, which achieves low OSNR penalty over a broad range of pulse rise-times. For 32-QAM, the optimum GI value is $5 \%$, but for higher orders (see in Figure 5c), it shifts to 10\%. Larger GI values introduce additional degradation on the performance of the system, which becomes more intense for low rise-times (see GI: 20 and 15\% in Figure 5c). Finally, we notice that the use of offset-QAM signals does not bring any gain in the performance of the switched channel. To this end, it is a much preferable strategy to operate with regular QAMs at the expense however of employing (optimized) guard-band values.

Different conclusions can be drawn, when analyzing the performance of the neighboring sub-carrier channel, see Figure 5(b) and (d). The case of zero GI was omitted from Figure 5(d) since it resulted in a rapid, extreme degradation of OSNR. Here, the existence of guard intervals is critical in keeping the OSNR penalty at low values for any rise-time. The larger the GI value, the broader the range of rise-times with low penalty performance. Also, the use of offset-QAMs against regular QAMs, is preferable only against zero guard-bands, whereas introducing a small guard-band can give significantly better performance. 


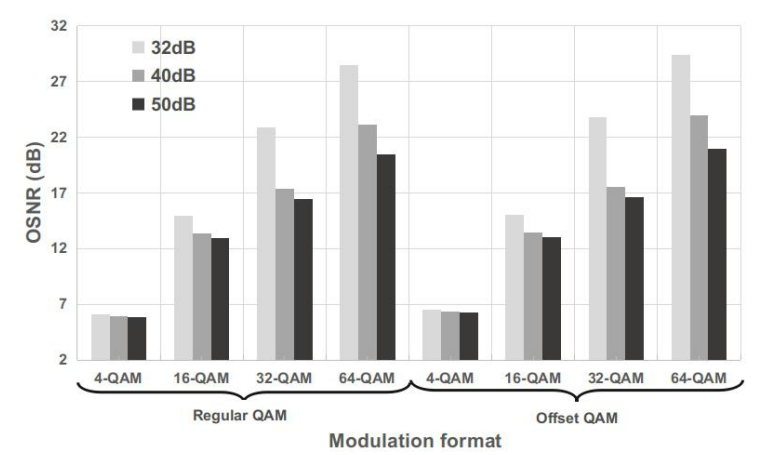

Fig. 6: Effect of gate contrast ratio in the required OSNR for achieving BER: $10^{-3}$ at the receiver.

Finally, we also studied node performance for different contrast ratios (CR) of the optical gates' switching window. These are commissioned to sample the input signal at the optimum point of their eye-diagram. Thus, non-ideal switching windows with a limited contrast ratio, degrade system performance. Here, a rectangular switching window is assumed with a varying contrast ratio, while calculating OSNR penalty for achieving $\mathrm{BER}=10^{-3}$ for the different constellation orders. Figure 6 displays the relevant results. To better demonstrate the effect of gate $\mathrm{CR}$ for the different formats, Figure 6 aggregates and displays the performance for the same CR vs the different modulation formats. Green bars correspond to a $30 \mathrm{~dB}$ contrast ratio, grey bars to $40 \mathrm{~dB}$ and blue to $50 \mathrm{~dB}$. When comparing the two modulation formats (regular and offset based QAM), in the case of $30 \mathrm{~dB}$ extinction, there is a penalty of $22.34 \mathrm{~dB}$ when upgrading from 4-QAM to 64-QAM. Almost the same penalty occurs for the offset QAM modulation $(22.84 \mathrm{~dB}$ from 4-offset QAM to 64-offset QAM). To this end, contrast ratio has little or no effect on the offset-based modulation format. When comparing same order modulation, the penalty in the OSNR varies and is summarized in the Table I. From Table I and Figure 6, it can be argued that the contrast ratio of the gate has little impact on the performance of offset based modulation formats at least for small constellation order and tops up at 0.9 $\mathrm{dB}$ for 64-QAM.

\section{Effect of FFT/i-FFT resolution}

Up to now, only ideal FFT/i-FFT filters had been considered in our numerical studies, having $\operatorname{sinc(fT)~frequency~response~}$ shape. However, the limited spectral resolution of a WSS switch, will have an imminent effect on the actual frequency response. Based on spatial filtering of the spectrally dispersed light, as occurring in Liquid Crystal on Silicon (LcoS) devices, and calculating the overlap integral, we identified the closest achievable approximation to the sinc(fT) function for various spectral resolution metrics (in GHz), defined as the spot size of a single frequency component (in $\mathrm{m}$ ) divided by the spatial dispersion (in $\mathrm{m} / \mathrm{Hz}$ )). A selection of frequency responses is reported in [14], where it is shown that resolutions greater than $5 \mathrm{GHz}$, (i.e.2/T) flatten out the sinc response, thus reducing the accuracy of the FFT. This in turns induces crosstalk, that degrades the overall performance.

In the proposed ROADM design, the impact of the limited spectral resolution on the add/drop performance of the node is
TABLE I: Calculated penalty in the required OSNR for the same order of modulation for different contrast ratios of the switching window

\begin{tabular}{|c|c|c|c|}
\hline \multicolumn{4}{|c|}{ OSNR penalty (dB) - add/drop Channel (Ch.4) } \\
\hline \multirow{2}{*}{$\begin{array}{l}\text { regular vs offset } \\
\text { QAM }\end{array}$} & \multicolumn{3}{|c|}{ Contrast Ratio } \\
\hline & $32 \mathrm{~dB}$ & $40 \mathrm{~dB}$ & $50 \mathrm{~dB}$ \\
\hline 4-QAM & -0.4 & -0.4 & -0.4 \\
\hline 16-QAM & -0.077 & -0.07 & -0.055 \\
\hline 32-QAM & -0.89 & -0.19 & -0.2 \\
\hline 64-QAM & -0.9 & -0.84 & -0.43 \\
\hline \multicolumn{4}{|c|}{ OSNR penalty (dB) - Adjacent Channel (Ch.3) } \\
\hline \multirow{2}{*}{$\begin{array}{l}\text { regular vs offset } \\
\text { QAM }\end{array}$} & \multicolumn{3}{|c|}{ Contrast Ratio } \\
\hline & $32 \mathrm{~dB}$ & $40 \mathrm{~dB}$ & $50 \mathrm{~dB}$ \\
\hline 4-QAM & 1.5 & 1.5 & 1.5 \\
\hline 16-QAM & 0.49 & 0.51 & 0,6 \\
\hline 32-QAM & 2.23 & 1.79 & 1.58 \\
\hline 64-QAM & 7 & 1.11 & -0.02 \\
\hline
\end{tabular}

depicted in Figure 7 (a) to (d). In particular, Figure 7 displays the sensitivity penalty for the added Channel 4 and its adjacent Channel 3 for regular (with and without a guard interval) and offset QAM signals. Results only for m=32,64 QAM formats are shown here, which have been obtained by selecting a gate contrast ratio of $30 \mathrm{~dB}$ and a 12 ps switching window. Figure 7(a) and (c) that correspond to the added Channel 4, show that for obtaining a reasonable penalty of less than $0.5 \mathrm{~dB}$ and for maintaining a $\mathrm{BER}=10^{-3}$ at the receiver, a resolution less than $1.5 \mathrm{GHz}$ is needed, [25]. For higher resolutions, the OSNR penalty rises rapidly reaching $3 \mathrm{~dB}$ for a $5 \mathrm{GHz}$ resolution. It worth's noting that employing guard interval (GI) increases the OSNR penalty faster. For example, for 64-QAM signals, a 15\% GI results in a $3 \mathrm{~dB}$ OSNR penalty for a $6 \mathrm{GHz}$ resolution while a $20 \% \mathrm{GI}$, in a $3 \mathrm{~dB}$ penalty for $3 \mathrm{GHz}$ resolution. In contrast, the offset QAM performance is not affected by the WSS spatial resolution. With respect to the adjacent Channel 3, results differ. Regular QAM formats affect less the node performance for a resolution smaller than $2 \mathrm{GHz}$. Further, the employment of a guard interval has little effect. However, for a resolution higher than $2 \mathrm{GHz}$, the required OSNR increases, and in particular for resolutions higher than $3 \mathrm{GHz}$, the offset QAM clearly outperforms and induces no further penalty. To this end, the increase of the FFT/i-FFT resolution has an imminent effect on OSRN penalty, due to the increasing ISI crosstalk induced on the added Channel 4.

With respect the performance of offset QAM modulation, this is almost constant, with no OSNR penalty. This is due to the negligible crosstalk induced from the QAM signals that pass through branch B and actually have no effect on the added channel. To this end, we may argue that the use of offset QAM is beneficial, whereas regular QAM modulated signals require a lower than $2 \mathrm{GHz}$ resolution (see Figure $7 \mathrm{~d}$ ) to achieve a similar (or even better) performance.

\section{CONCLUSION}

In this work, a ROADM architecture is proposed that can accommodate both regular and offset based QAM signals and enable add/drop operations at a granularity level of a single optical sub-channel of OFDM super-channels. Albeit the proposed ROADM design requires specialized optical 

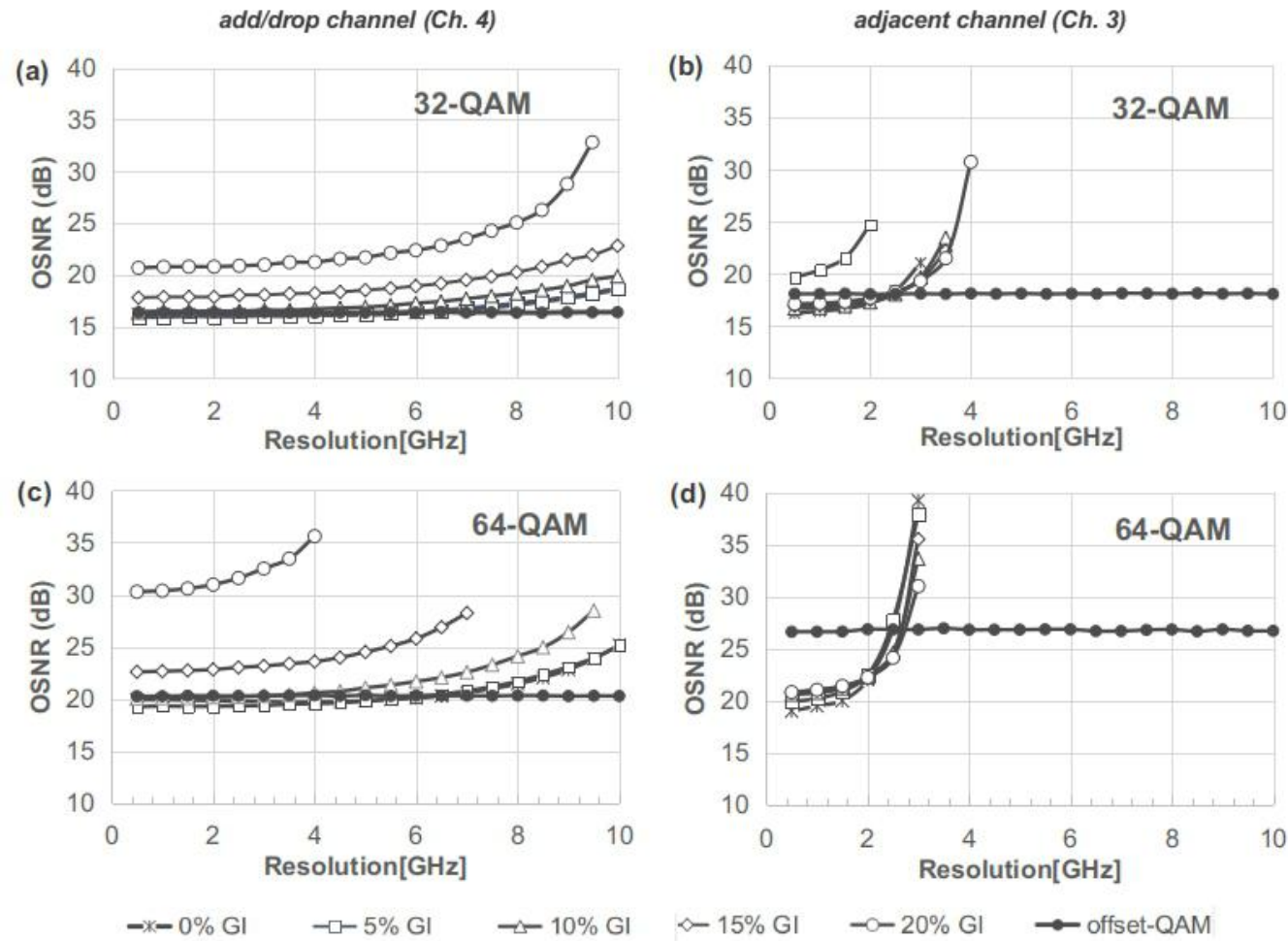

Fig. 7: Effect of the WSS resolution that implement the FFT/i-FFT filtering in the node as well as the receiving end. Required OSNR at the receiver for achieving BER: $10^{-3}$ for different constellation high-order (32 and 64-QAM) signal of the add-drop Channel 4 and its closest neighboring Channel 3. Results have been taken for different guard interval for both the regular as well as the offset QAM format.

hardware and its implementation is challenging for high order OFDM signals, it provides a clear path implementing an interferonetric structure that relies on purely all-optical processes, i.e. FFT/i-FFT filtering and time domain sampling. Also, it is modular, in the sense that that additional interferometer sub-paths can be added to expand ROADM operation to higher sub-channel counts, taking advantage of commercial WSSs that also bear a large number of ports. In this work, we have investigated and optimize several sub-system parameters as for example pulses rising time, size of the guard interval used, power input levels and gate contrast ratio. Numerical studies have shown that offset QAM modulation is beneficial against regular-QAM modulated sub-channels with zero guards intervals, especially at high constellation orders.

The results demonstrated a scaling capability for up to 64-QAM signals, whereas for larger constellation orders the performance was primarily limited by the finite rise time of the optical pulses. Furthermore, operating with offset QAM signals or a short guard-band interval $(10 \%)$, proved to be the most preferable choice in terms of performance.

\section{ACKNOWLEDGMENT}

The authors would like to acknowledge Prof. Dan Marom for sharing his simulation code on the transfer function of the LcoS based photonic spectral processor.

\section{REFERENCES}

[1] S. Sygletos, I. Tomkos, J. Leuthold, "Technological challenges on the road towards transparent networking," in Journal of Optical Networking, vol. 7, no. 4, pp. 321-350, April 2008.

[2] A. D. Ellis, F. C. Gunning, "Spectral density enhancement using coherent WDM," IEEE Photon. Technol. Lett., vol. 15, no. 2, pp. 504-506, Jan. 2005

[3] A. Sano et al., "No-Guard-Interval Coherent Optical OFDM for 100-Gb/s Long-Haul WDM Transmission," IEEE Journal of Lightwave Technology, vol. 27, no. 16, pp. 3705-3713, Aug.15, 2009. doi:10.1109/JLT.2009.2023369

[4] R. Schmogrow, M. Winter, M. Meyer, D. Hillerkuss, S. Wolf, B. Baeuerle, A. Ludwig, B. Nebendahl, S. Ben-Ezra, J. Meyer, M. Dreschmann, M. Huebner, J. Becker, C. Koos, W. Freude, and J. Leuthold, "Real-time Nyquist pulse generation beyond $100 \mathrm{Gbit} / \mathrm{s}$ and its relation to OFDM," Optics Express vol. 20, no. 1, pp. 317-337, Dec. 2012

[5] K. Igarash et al., "1.03-Exabit/s $\mathrm{km}$ super-Nyquist- WDMtransmission over 7,326-km sevencore fiber," presented at the 39th European Conference on Optical Communications, London, U.K., 2013, Paper PD3.E.3

[6] R. Rudnick, A. Tolmachev, D. Sinefeld, O. Golani, S. Ben-Ezra, M. Nazarathy, D. M. Marom, "Sub-banded / single-sub-carrier drop-demux and flexible spectral shaping with a fine resolution photonic processor." at the European Conference on Optical Communication (ECOC), Sept. $21-25,2014$

[7] Bill Corcoran et al., "Multipass Performance of a Chip-Enhanced WSS for Nyquist-WDM Sub-Band Switching", IEEE Journal of Lightwave Technology, vol. 34, no. 8, pp. 1824 -1830, April 2016 
[8] Peter J. Winzer, "An Opto-Electronic Interferometer and Its Use in Subcarrier Add/Drop Multiplexing", IEEE Journal of Lightwave Technology, vol. 31, no. 11, pp. 1775-1782, June 2013

[9] T. Richter et al., "Coherent Subcarrier Processing Node Based on Optical Frequency Conversion and Free-Running Lasers," IEEE Journal of Lightwave Technology, vol. 33, no. 3, pp. 685-693, Feb.1, 12015. doi: 10.1109/JLT.2014.2379472

[10] C. Schmidt-Langhorst et al., "Coherent Optical Subcarrier Processing and Add/Drop Multiplexing," at the European Conference on Optical Communication (ECOC), Sept. 18-22, 2016

[1 1] T. Kato et al., "Coherent-Optical In-Line Add/Drop of PDM Tributaries of Subcarrier Multiplexed Signals", IEEE Photonics Technology Letters, vol. 28 , no. 13 , pp. 1465-1468, July 2016

[12] E. Pincemin et al., "Multi-band OFDM transmission at 100 Gbps with sub-band optical switching," IEEE Journal of Lightwave Technology vol. 32, no. 12, pp. 2202-2219, May 2014

[13] A. Sano et al., "No-Guard-Interval Coherent Optical OFDM for 100-Gb/s Long-Haul WDM Transmission," IEEE Journal of Lightwave Technology, vol. 27, no. 16, pp. 3705-3713, Aug.15, 2009. doi: $10.1109 /$ JLT.2009.2023369

[14] S. Sygletos et al., "Numerical investigation of all-optical add-drop multiplexing for spectrally overlapping OFDM signals," Optics Express, vol. 23, no. 5, pp. 588-5897, Feb. 2015.

[15] D. Monogios, K. Vlachos, F. Ferreira and S. Sygletos, "Sub-channel add/drop multiplexing in offset-QAM OFDM signals", in proceeding of Photonics in Switching 2018, Sept. 19 - 21, 2018.

[16] J. Zhao, A. D. Ellis, "Offset-QAM based coherent WDM for spectral efficiency enhancement," Optics Express, vol. 19, no. 5, pp. 14617, Jul. 2011.

[17] S. J. Fabbri, S. Sygletos, A. Perentos, E. Pincemin, K. Sugden and A. D. Ellis, "Experimental Implementation of an All-Optical Interferometric Drop, Add, and Extract Multiplexer for Superchannels," IEEE Journal of Lightwave Technology, vol. 33, no. 7, pp. 1351-1357, April 2015

[18] D. Hillerkuss, M. Winter, M. Teschke, A. Marculescu, J. Li, G. Sigurdsson, K. Worms, S. Ben Ezra, N. Narkiss, W. Freude, J. Leuthold, "Simple all-optical FFT scheme enabling Tbit/s real time signal processing," Optics Express, vol. 18, no. 9, pp. 9324-9340, April 2010

[19] A. J. Lowery, "Design of arrayed-waveguide grating routers for use as an optical OFDM demultiplexers," Opt. Express vol. 18, no. 13, pp. 14129-14143, Jun. 2010

[20] S. Kodama, T. Ito, N. Watanabase, S. Kondo, H. Takeuchi and T. Ishibashi, "2.3 picoseconds optical gate monolithically integrating photodiode and electro-absorption modulator," Electron. Lett., vol. 37, no. 19, pp. 1185-1186, Sep. 2001

[21] K. E. Stubkjaer, "Semiconductor optical amplifier-based all-optical gates for high-speed optical processing," IEEE Journal of Selected Topics in Quantum Electronics, vol. 6, no. 6, pp. 1428-1435, Nov.-Dec. 2000. doi: 10.1109/2944.902198.

[22] D. M. Marom, D. T. Nelson, D. S. Greywall, C. S. Pai, N. R. Basavanhally, V. A. Aksyuk, D. O. Lopez, F. Pardo, M. E. Simon, Y. Low, P. Kolodner, and C. A. Bolle, "Wavelength-selective 1xK switches using free-space optics and MEMS micromirrors: theory and implementation," IEEE J. Lightwave Technol., vol. 23, no. 4, pp. 1620-1630, April. 2005

[23] D. Sinefeld, D. M. Marom, "Hybrid guided-wave/free-space optics photonic spectral processor based on LcoS phase only modulator," IEEE Photon. Technol. Lett., vol 22, no. 7, pp. 510-512, April 2010.

[24] M. C. Jeruchim, "Techniques for estimating the bit error rate in the simulation of digital communication systems," IEEE J. Selec. Areas Commun., vol 2, no. 1, pp. 153-170, (1984).

[25] Dan M. Marom; Roy Rudnick; Noam Goldstein; Ori Golani; David Sinefeld, "Realization of sub-1 GHz resolution photonic spectral processors for flexible optical networks", in Proc. ECOC 2015 (Valencia)

Kyriakos G. Vlachos received his Dipl.-Ing. degree in electrical and computer engineering from the National University of Athens (NTUA), Greece, in 1998 and his Ph.D. in electrical and computer engineering, also from NTUA, in 2001. From 1997 to 2001 he was a Senior Research Associate in the Photonics Communications Research Laboratory (ICCS/NTUA). In April 2001 he joined
Bell Laboratories, Lucent Technologies, working on behalf of the Applied Photonics Group. Prof. Vlachos conducted research on high-speed optical networks and DWDM transmission techniques. During 2003, he joined the National Regulation Authority of Telecommunication andPostal Service of Greece (EETT), where he served as a Scientific Advisor for various techno-economic issues for the promotion of broadband technologies. Since 2003, he was also a member of the Computer Engineering Laboratory of the Technical University of Delft,and since 2005 he has been a Faculty Member of the Computer Engineering and Informatics Department of the University of Patras, Greece. Prof. Vlachos has participated in various research projects funded by the European Commission (IST-STOLAS, IST-PRO3, ESPRIT-DOALL, ephoton/ONe, ICT-BONE, and ICT-DICONET). He periodically acts as a scientific reviewer for the General Secretariat for Research and Technology of Greece (GSRT) as well as for the European Commission and the Netherlander Organization for Scientific Research, Technology Foundation. Prof. Vlachos is the (co)author of more than 100 journal and conference publications and holds 2 patents.

Stylianos S. Sygletos received his Dipl-Ing, degree in electrical and computer engineering from the National University of Athens (NTUA), Greece, in 2000 and his Ph.D. in electrical and computer engineering, also from NTUA, in 2006 In March 2006 he joined Athens Information Technology Centre where he worked on impairment constraint based routing and modelling of quantum dot semiconductor optical amplifiers (QD-SOAs). In 2007-2009 he was a postdoctoral researcher in University of Karlsruhe continuing his work on QD-SOAs. In 2009-2012, he worked as a researcher in Tyndall National Institute in the field of Phase Sensitive Amplifiers for regeneration applications and the development of suitable optical phase locking schemes. Since 2012 he is a senior research fellow and principal investigator in Aston Institute of Photonics Technologies. He is an (co)-author of more than 150 publications in journals and conferences and holds 4 patents. 